ARTICLE

https://doi.org/10.1038/s41467-019-09052-w

\title{
Genetic dissection of Nodal and Bmp signalling requirements during primordial germ cell development in mouse
}

\author{
Anna D. Senft ${ }^{1}$, Elizabeth K. Bikoff ${ }^{1}$, Elizabeth J. Robertson ${ }^{1} \&$ Ita Costello ${ }^{1}$
}

The essential roles played by Nodal and Bmp signalling during early mouse development have been extensively documented. Here we use conditional deletion strategies to investigate functional contributions made by Nodal, Bmp and Smad downstream effectors during primordial germ cell (PGC) development. We demonstrate that Nodal and its target gene Eomes provide early instructions during formation of the PGC lineage. We discover that Smad2 inactivation in the visceral endoderm results in increased numbers of PGCs due to an expansion of the PGC niche. Smad1 is required for specification, whereas in contrast Smad4 controls the maintenance and migration of PGCs. Additionally we find that beside Blimp1, down-regulated phospho-Smad159 levels also distinguishes PGCs from their somatic neighbours so that emerging PGCs become refractory to Bmp signalling that otherwise promotes mesodermal development in the posterior epiblast. Thus balanced Nodal/Bmp signalling cues regulate germ cell versus somatic cell fate decisions in the early posterior epiblast.

\footnotetext{
${ }^{1}$ Sir William Dunn School of Pathology, University of Oxford, Oxford OX1 3RE, UK. Correspondence and requests for materials should be addressed to E.J.R. (email: elizabeth.robertson@path.ox.ac.uk)
} 
P rimordial germ cells (PGCs), the precursors of sperm and eggs, are initially detectable in the early mouse embryo at around embryonic day (e) 6.25, prior to the onset of gastrulation ${ }^{1}$. Early fate mapping experiments revealed that the proximal posterior epiblast (PPE) gives rise to both the extraembryonic mesoderm (ExM) and PGC cell populations ${ }^{2}$. The regulatory signals governing these cell fate decisions remain illdefined. The PR domain containing zinc finger transcription factor Blimp1 (encoded by Prdm1) plays an essential role during PGC specification and expression at e6.25 identifies precursor PGCs (pre-PGCs) ${ }^{3,4}$. Commitment to the PGC lineage becomes evident slightly later between e6.75 and e7.5 when pre-PGCs activate expression of germ cell markers such as Stella (Dppa3) and Ap2 $\gamma$ (Tfap2c), concomitantly reactivate expression of pluripotency genes, including Sox2 and repress somatic gene expression $^{1,5}$. By e8.5, specified PGCs have migrated from the base of the allantois into the overlying endoderm, and subsequently migrate along the dorsal hindgut endoderm before homing to and colonising the genital ridges from e10.5 ${ }^{1}$. Extensive chromatin remodelling and genome-wide epigenetic reprogramming occurs during migration ${ }^{6}$. Transcriptional profiling experiments analysing PGCs in vivo and in vitro differentiated PGC-like cells (PGCLCs) have provided insights into the dynamic transcriptional changes that accompany PGC maturation $5,7,8$.

Correct patterning of the early post-implantation stage embryo depends on reciprocal signalling cues by members of the TGF $\beta$ family of secreted growth factors controlling cell-cell interactions between the pluripotent epiblast and the overlying extraembryonic tissues namely the extra-embryonic ectoderm (ExE) and the visceral endoderm $(\mathrm{VE})^{9,10}$. Temporally and spatially restricted expression of Nodal and Bmp ligands results in activation of their cognate receptors, phosphorylation of the intracellular effectors, Smad2 and Smad3 (Smad23), or Smad1, Smad5 and Smad9 (Smad159), respectively, that associate with the coSmad Smad4, translocate to the nucleus and activate cell-type specific target gene expression ${ }^{11}$. Nodal signalling in the early epiblast is required for correct patterning of the VE, formation of the anterior-posterior (A-P) axis and also promotes development of the $\mathrm{ExE}^{12}$. Together with Bmp signals from the ExE, Nodal initiates mesoderm induction and primitive streak (PS) formation within the PPE 12,13 . During gastrulation, graded Nodal signals pattern the $\mathrm{PS}^{10}$. Thus, highest levels of Nodal signalling are required for specification of anterior PS derivatives. Lowering Nodal expression levels in the PS, or depleting Smad23 in the epiblast results in failure to correctly specify the anterior definitive endoderm (DE) and the embryonic midline ${ }^{14}$.

In contrast $\mathrm{Bmp} / \mathrm{Smad} 159$ signals promote the formation of ExM. Loss of Bmp4 from the ExE disrupts gastrulation and is associated with truncation of posterior structures, including the allantois and yolk sac ${ }^{13}$. Both Smad1 and Smad5 null embryos also display ExM tissue defects ${ }^{15,16}$. Mutant embryos lacking Bmp4 or Bmp8b expression in the ExE or those lacking Bmp2 in the VE display compromised PGC development in the epiblast $^{17-19}$. Bmp4 null embryos entirely lack mature PGCs, while Bmp4 heterozygous embryos contain reduced numbers of PGCs. The observation of reduced numbers of PGCs in embryos lacking either Smad1 or Smad515,16, as well as in Smad1/5 double heterozygous embryos ${ }^{20}$, provides convincing evidence that dosedependent Bmp signalling governs PGC specification. The Wnt signalling pathway also regulates PGC development ${ }^{21}$. Wnt3 is normally induced in the posterior epiblast in response to Nodal/ Bmp signalling 22 . Wnt 3 mutants fail to gastrulate ${ }^{23}$ and lack a detectable pre-PGC population ${ }^{21}$. Collectively, these findings demonstrate that patterning during gastrulation and PGC formation are co-ordinately regulated by dynamic signalling events. However, given the complex morphological disturbances observed in loss of function mutant embryos, it has proven difficult to further dissect the crosstalk between the embryonic and extra-embryonic tissues. In particular since Nodal null embryos arrest prior to gastrulation ${ }^{24}$, any possible role of Nodal signalling during PGC specification remains to be explored.

Here, we exploit tissue specific conditional deletion strategies to investigate functional contributions made by Nodal and Bmp signalling within the embryonic versus extra-embryonic tissues. We directly assess the distinct roles played by various signalling pathway components in the VE and the epiblast during formation of the PGC lineage. Collectively, our experiments provide insights into the signalling cues that cooperatively regulate the size of the founding pre-PGC population and govern the PGC developmental programme during induction, specification and migration at early post-implantation stages of mouse development.

\section{Results}

Smad2 in the VE restricts Bmp signals to the proximal region. In Smad2 null embryos, lacking the anterior VE (AVE) signalling centre, the epiblast adopts an exclusively ExM fate ${ }^{12,25}$. These mis-patterned embryos arrest at around e9.5. Here, to examine cell-type specific Smad2 functional contributions within the VE, we crossed animals carrying a conditional Smad2 allele (Smad2CA) with heterozygous Smad2 $2^{+/-}$mice carrying the TtrCre transgene ${ }^{26}$ (Supplementary Figure 1A). As shown below, we found that the Smad2 $\Delta$ VE embryos phenocopy the Smad2 $2^{-I-}$ embryos, strengthening the idea that the dramatic tissue disturbances observed in the null embryos predominantly reflect the loss of Smad2 signalling in the AVE.

To assess the possible impact on Bmp signalling, we analysed phospho-Smad159 (p-Smad159) localisation. At e5.5 p-Smad159 expression is normally restricted to the proximal VE. However, pSmad159 staining in Smad2 $\Delta$ VE e5.5 embryos is detectable throughout the entire VE, including the distal region (Fig. 1a). Whole-mount in situ hybridisation experiments demonstrate that Bmp4 expression remains unchanged, whereas Smad2 $\Delta \mathrm{VE}$ embryos lack Bmp2 transcripts (Supplementary Figure 1B). Thus, higher levels of p-Smad159 cannot simply be explained due to increased expression of Bmp ligands.

Antagonistic Bmp and Nodal signalling cues govern VE specification ${ }^{9}$. However, the regulatory mechanisms that normally restrict p-Smad159 signalling to the proximal VE have yet to be fully characterised. The TGF $\beta$ antagonist Gdf3, expressed in the epiblast and distal VE, directly antagonises Bmp4 activity ${ }^{27,28}$. Moreover, selective mesoderm expansion in double homozygous embryos lacking both $G d f 3$ and the closely related ligand $G d f 1$ has been documented ${ }^{29}$. Here, we observe in Smad2 $\Delta \mathrm{VE}$ embryos that Gdf3 expression is absent in the VE and reduced in the epiblast (Fig. 1b). Thus, up-regulated p-Smad159 activity in Smad2 $\Delta V E$ embryos potentially reflects decreased Gdf3 expression levels.

Loss of Smad2 from the VE expands the PGC niche. Alkaline phosphatase (AP) positive presumptive PGC clusters were previously identified in e8.5 Smad2 $2^{-1-}$ embryos ${ }^{15}$. PGCs are formed within the PPE and are reliant on Bmp signalling from the adjacent ExE for their development. To evaluate if and when PGCs are formed in Smad2 $\Delta$ VE embryos, where there is an excess of Bmp signalling, we examined PGC marker gene expression. Nanog, normally reactivated in the early proximal epiblast $^{30}$, is also strongly expressed in developing PGCs ${ }^{31}$. As expected in control embryos, we detected cells co-expressing Nanog and the pluripotency marker Oct4 in the PPE (Fig. 1c). Similarly, at e6.5 Smad2 $\Delta \mathrm{VE}$ embryos contain Nanog/Oct4 
a
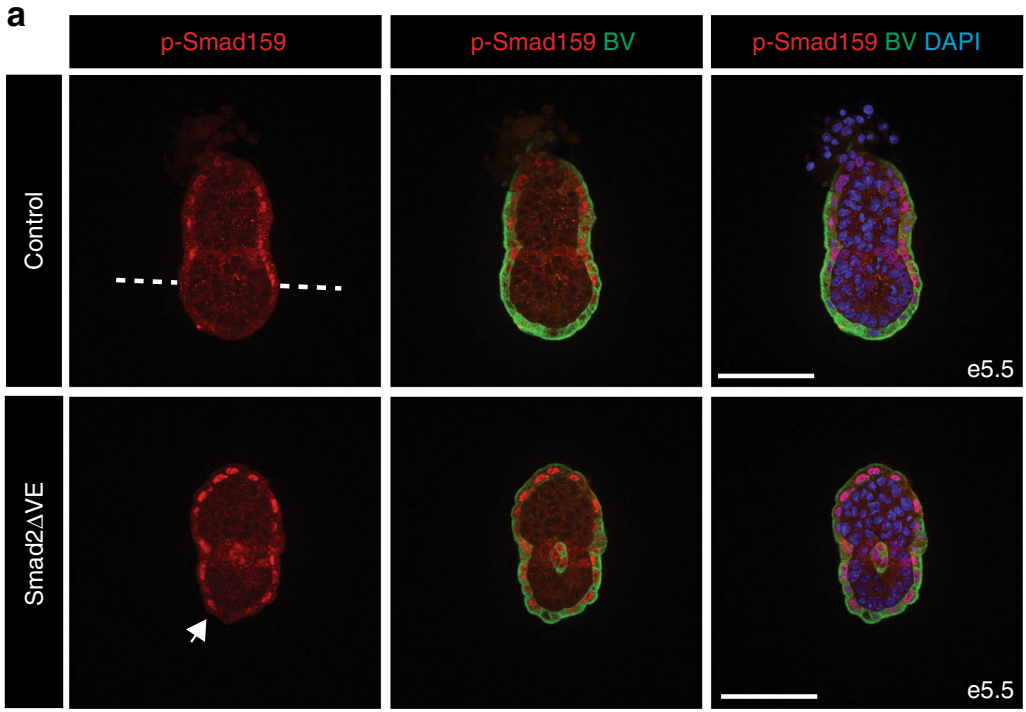

b

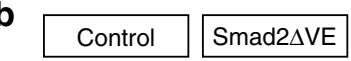

C
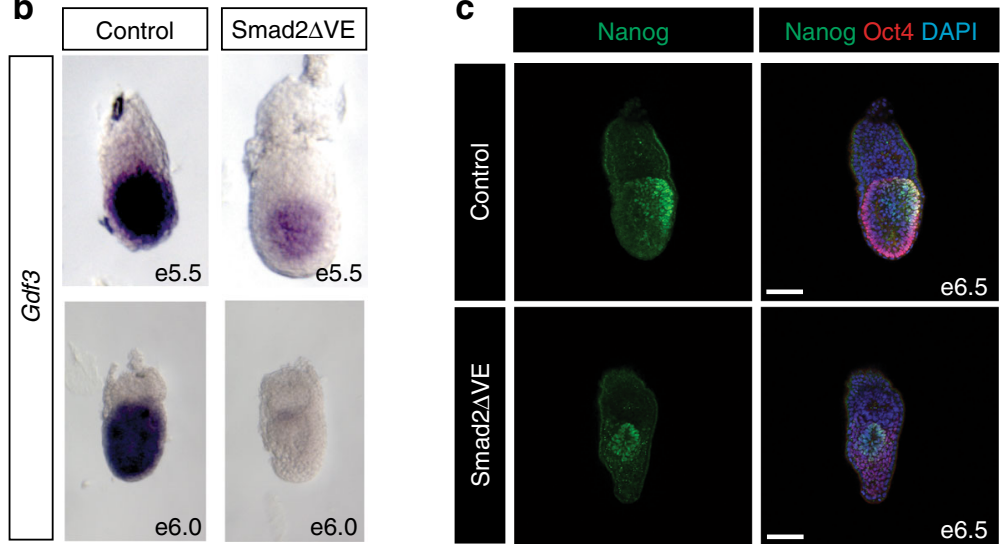

d
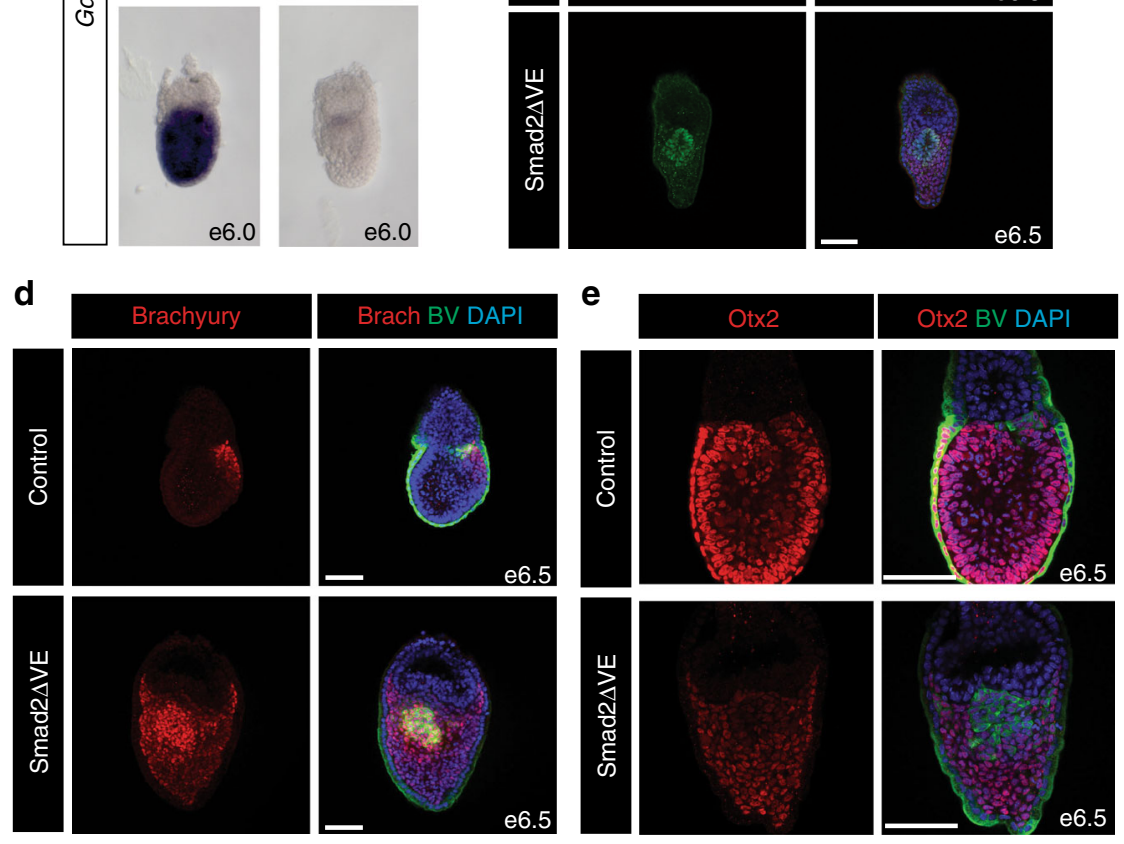

Fig. 1 Imbalanced Bmp/Nodal signalling and expansion of the PGC niche caused by Smad2 inactivation in the VE. a Representative images of p-Smad159 immunofluorescence (IF) staining of e5.5 embryos carrying the Blimp1-mVenus (BV) transgene, counter stained with DAPI. Dashed line indicates extent of proximal p-Smad159 staining in control embryos. The arrow indicates expanded p-Smad159 in the distal VE of Smad2 $\Delta$ V embryos. b Whole-mount in situ hybridisation analysis of Gdf3 expression in control and Smad2 $\Delta V E$ embryos at e5.5 and e6.0. c Nanog and Oct4 co-staining in e6.5 control and Smad2 $\Delta$ VE embryos. d Brachyury IF in e6.5 control and Smad2 $\Delta$ VE BV-expressing embryos. e Otx2 staining and BV expression at e6.5. All IF staining images were counter stained with DAPI. Scale bars $=100 \mu \mathrm{m}$

double positive cells adjacent to the ExE, but these were located in a central position in the epiblast (Fig. 1c).

Next, to assess whether these cells correspond to pre-PGCs, we used the membrane tethered Blimp1-mVenus (BV) BAC transgene that faithfully recapitulates Blimp1 expression in both the VE and the developing $\mathrm{PGCs}^{32}$. Smad2 $\triangle \mathrm{VE}$ mutants expressing the $\mathrm{BV}$ transgene clearly contain $\mathrm{BV}$-positive $\left(\mathrm{BV}^{+}\right)$ pre-PGCs that also co-express E-Cadherin (Supplementary Figure 1C). As judged by immunohistochemistry these cells strongly express endogenous Blimp1 protein (Supplementary Figure 1D). Interestingly, in Smad $2 \Delta \mathrm{VE}$ embryos $\mathrm{BV}^{+}$pre-PGCs are initially detectable within the epiblast at e5.5, $12-18 \mathrm{~h}$ before 
their appearance in wild-type embryos (Fig. 1a). Slightly later at e6.5, the Smad $2 \Delta V E$ proximal epiblast contains increased numbers of $\mathrm{BV}^{+}$cells as compared to control wild-type embryos (Fig. 1d, e).

$\mathrm{BV}^{+}$cells in the proximal epiblast at e6.5 normally express Brachyury and retain E-Cadherin expression. In contrast the adjacent mesodermal cells also strongly express Brachyury but down-regulate E-Cadherin expression (Fig. 1d, Supplementary Figure 1C). The central core of $\mathrm{BV}^{+}$epithelial cells in Smad $2 \Delta \mathrm{VE}$ embryos is likewise surrounded by Brachyury positivemesodermal cells (Fig. 1d). At e6.5, in both control and Smad2 $\Delta$ VE embryos $\mathrm{BV}^{+}$cells weakly express Otx2, Eomes and Sox2 (Fig. 1e, Supplementary Figure 1E and F). Slightly later at e7.5 $\mathrm{BV}^{+}$cell clusters retain E-Cadherin and co-express Stella as well as Oct4 (Supplementary Figure $1 \mathrm{G}$ and $\mathrm{H}$ ). Overall, in Smad2 $\Delta$ VE embryos we observe increased numbers of BV, Oct4, Nanog and Stella co-expressing PGCs surrounded by Brachyurypositive, E-Cadherin-negative mesodermal cells. Thus, Smad2 expression in the VE normally restricts expansion of the PGC niche so that only a small subset of posterior epiblast cells become allocated to a PGC fate.

Next, to examine Smad2 functional contributions within the epiblast we made use of the Sox2-Cre deleter strain ${ }^{33}$. Stella ${ }^{+}$ PGCs are readily detectable at e8.5 in Smad2 $\Delta$ Epi embryos, suggesting that Smad2 is dispensable for PGC specification (Supplementary Figure 2A). However, it seems likely that the closely related Smad3 effector, known to be robustly expressed in the epiblast ${ }^{14}$, functionally compensates.

Blimp1 is strongly expressed in the VE, but experiments to date have failed to demonstrate any VE-specific functional contributions. To test whether Blimp1 VE expression may influence PGC specification, we used the Ttr-Cre deleter strain. As shown in Supplementary Figure 2C, selective loss of Blimp1 in the VE has no noticeable effect on the formation of Blimp1-expressing PGCs at the base of the allantois (Supplementary Figure 2B and C). Moreover, Prdm $1 \Delta$ VE mice are viable and fertile (Supplementary Table 1).

Both Nodal and Eomes are essential during PGC specification. Smad 2 and the closely related Smad 3 are intracellular effectors of the Nodal pathway. Nodal null ES cells, as well as Smad23 double mutant ES cells, fail to generate PGCLCs in vitro ${ }^{34,35}$. To investigate Nodal functional contributions in vivo we examined formation of pre-PGCs in $\mathrm{Nodal}^{-1-}$ embryos. At e5.5 Nanog expression levels are dramatically reduced as compared to control embryos (Fig. 2a, Supplementary Figure 2D). However, Nodal ${ }^{-1-}$ embryos robustly express the epiblast marker Oct4 (Supplementary Figure 2D). Interestingly, $\mathrm{Nodal}^{-1-}$ embryos display precocious induction of $\mathrm{BV}^{+}$epiblast cells (Fig. 2a). These $\mathrm{BV}^{+}$cells have either weak or no Nanog expression at e5.5 (Fig. 2a). By e6.5 there is no expression of Nanog in the $\mathrm{BV}^{+}$epiblast cells or indeed any other cells of the epiblast (Supplementary figure 2E). At e6.5 the expanded $\mathrm{BV}^{+}$cell population in $\mathrm{Nodal}^{-1-}$ embryos lack precocious expression of specified PGC markers, such as Ap2 $\gamma$ (Supplementary Figure 2F), even though the BV transgene was prematurely expressed. Notably at e6.5 Nodal mutants do not maintain Bmp4 expression ${ }^{12}$, hence an important germ cell inducing signal is also absent in these embryos. Accordingly, at e7.5 Ap2 $\gamma / \mathrm{BV}^{+}$co-expressing cells are not observed in Nodal $^{-1-}$ embryos (Supplementary Figure 2G). Hence, specified PGCs are not observed in Nodal $^{-1}$ - embryos. As for Smad2 $\Delta$ VE embryos, e5.5 Nodal mutants similarly exhibit increased levels of pSmad159 staining in the distal VE (Fig. 2b). Moreover p-Smad159 is also detectable throughout the epiblast (Fig. 2b). These results suggest that Nodal functions in vivo to promote optimal levels of
Nanog during pre-PGC development and also to spatially restrict the PGC niche.

The T-box transcription factor Eomes, acting downstream of Nodal signalling, plays essential roles during gastrulation ${ }^{36,37}$. Eomes is also required to pattern the $\mathrm{VE}^{38}$. As shown in Supplementary Figure $2 \mathrm{H}$, Eomes $\Delta$ VE embryos contain Blimp1 ${ }^{+}$ epiblast cells. Thus, Eomes activity in the VE is non-essential for Blimp1 induction in the epiblast. Conditional deletion in the epiblast (Eomes $\Delta$ Epi) results in defective epithelial-tomesenchymal transition (EMT) and the failure of nascent mesoderm cells to down-regulate E-Cadherin and exit the PS $^{36}$. Eomes is expressed in early pre-PGCs, but becomes downregulated by late streak stages (Supplementary Figure 1E, Supplementary Figure 2I). Interestingly, Eomes $\Delta$ Epi e7.5 embryos contain an expanded epithelial-like $\mathrm{BV}^{+}$cell population (Fig. 2c) that also express endogenous Blimp1 protein (Supplementary Figure $2 \mathrm{H}$ ). Nanog expression is reactivated within the epiblast, however, the $\mathrm{BV}^{+}$cell population in Eomes $\Delta \mathrm{Epi}$ embryos is largely Nanog negative (Fig. 2d). Moreover, at e7.5 $\mathrm{BV}^{+}$cells fail to activate the PGC markers Ap2 $\gamma$, Sox 2 and Stella (Fig. 2e) and only a subset retain Brachyury activity (Fig. 2f). These results demonstrate that the loss of the T-box transcription factor Eomes in the epiblast disrupts the PGC developmental programme in vivo.

Smad1/4 roles in PGC specification and maintenance or migration. Smad1 is an intracellular effector of the Bmp signalling pathway, while the co-Smad, Smad4, is important for transducing aspects of both Nodal and Bmp signalling. Smad1 and Smad4 have previously been implicated in PGC development, as both Smad1 null and Smad4 $\Delta$ Epi embryos lack AP-positive migrating PGCs at e8.5 15,39 . However, cooperative or possibly unique functional roles at distinct developmental stages during PGC lineage specification have yet to be examined. Initially to explore Smad4 functional requirements in the VE, we generated Smad $4 \Delta$ VE embryos. We found at e7.5 that Blimp1-expressing PGCs are formed appropriately at the base of the allantois (Supplementary figure 3A). Similarly, Smad4 activity in the epiblast is non-essential for PGC specification. BV/Stella coexpressing cells are detectable on the posterior side of Smad4$\Delta$ Epi embryos at e7.5 (Supplementary figure 3B). However, at e8.5 these Stella/ $\mathrm{BV}^{+}$PGCs remain at the base of the allantois and fail to migrate towards the gut endoderm (Fig. 3a). In addition, we used a Blimp1-Cre deleter strain ${ }^{40}$ to selectively eliminate Smad4 expression within the pre-PGC cell population (Smad4 $\triangle$ PGC). Relatively, few Stella expressing cells were present in e8.5 Smad4 $\triangle$ PGC embryos (Supplementary Figure 3C). These results demonstrate that Smad4 is dispensable for initial PGC specification, but is required for PGC maintenance and/or migration.

Smad $1^{-1-}$ embryos form only a rudimentary allantoic bud and develop a distinctive out-pocketing of the proximal posterior $\mathrm{VE}^{15}$. At e7.5, only a few BV/Oct $4^{+}$pre-PGCs are detectable underneath the ruffled VE (Fig. 3b) and mature Stella positive PGCs fail to emerge ${ }^{15}$. To further investigate Smad1 functional requirements in the $\mathrm{VE}$, we used the Ttr-Cre deleter strain. Smad1 $\triangle$ VE embryos display extensive ruffling of the VE (Supplementary Figure 3D). However, Blimp1 ${ }^{+}$pre-PGCs are occasionally observed in the early epiblast (Supplementary Figure 3E). An epiblast specific Smad1 deletion likewise results in $\mathrm{VE}$ ruffling and the appearance of rare $\mathrm{BV} /$ Oct4 double positive cells underneath the overgrown VE (Fig. 3c). However, as for Smad1 null embryos these $\mathrm{BV} / \mathrm{Oct} 4^{+}$cells fail to form specified PGCs. The ability to generate some BV/Oct $4^{+}$cells may be due to Smad5 compensation, which is also expressed in the 
a
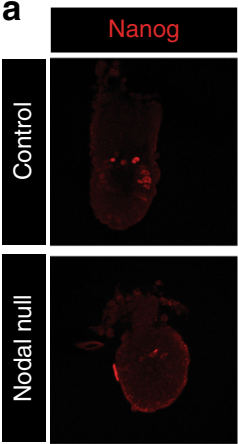

C
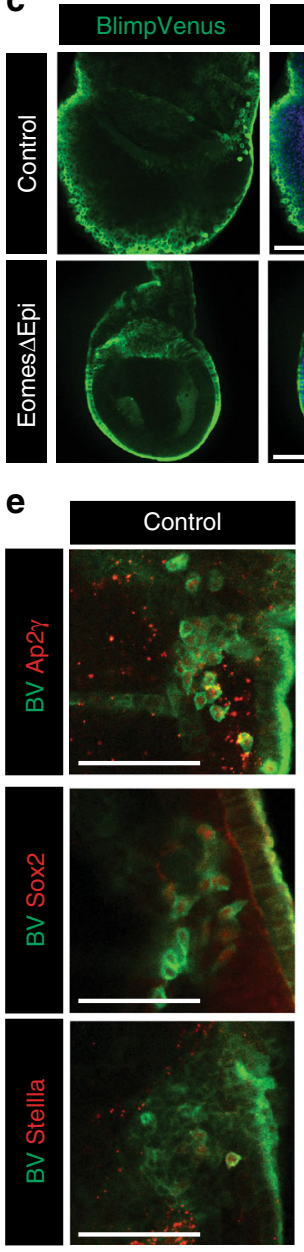
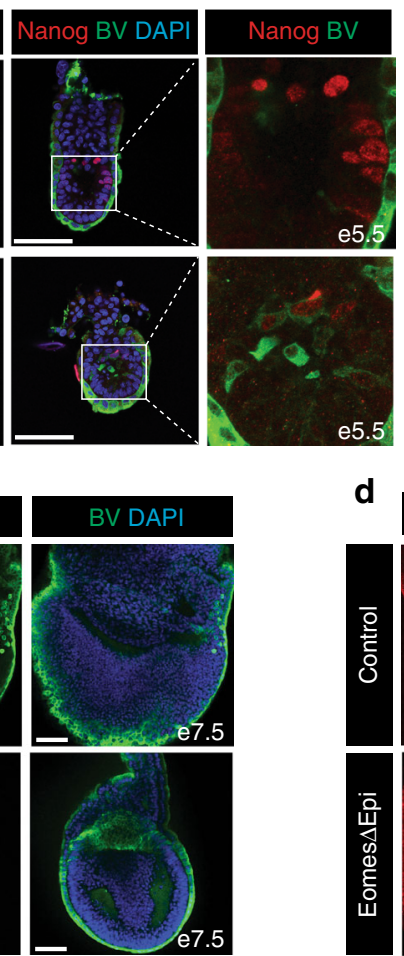

e5.5
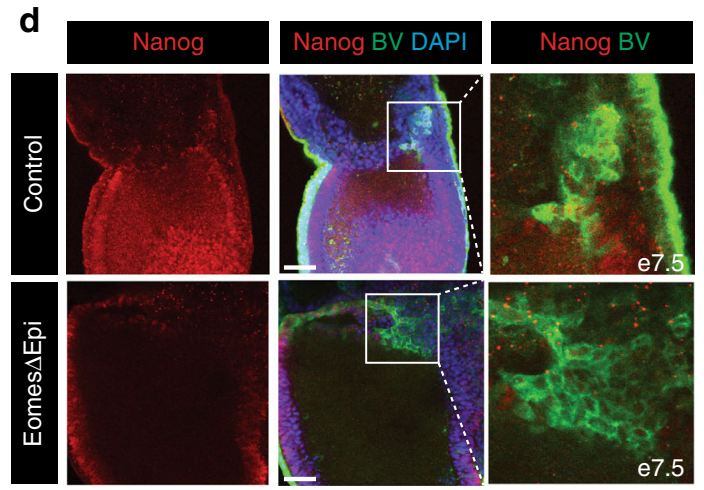
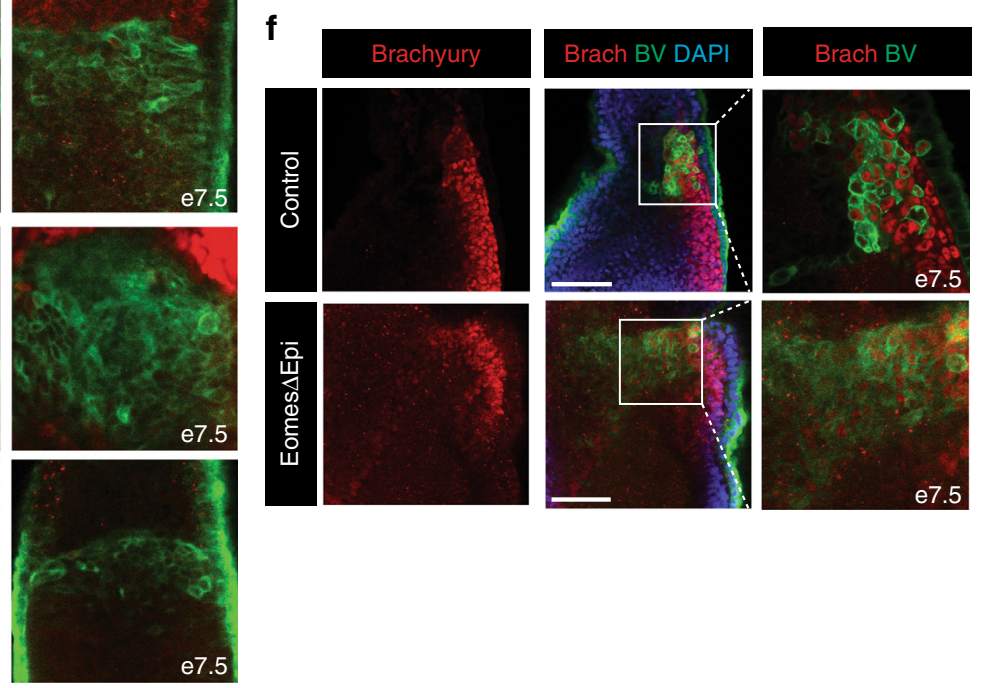

Fig. 2 Nodal and its downstream target Eomes regulate early stages of PGC development. a Reduced levels of Nanog expression in e5.5 Nodal null BV ${ }^{+}$ embryos. b Nodal null BV+ embryos display an expansion of p-Smad159 staining in the distal VE, as indicated by arrows. Arrowhead indicates lowered pSmad159 staining within $\mathrm{BV}^{+}$cells. $\mathbf{c}$ Expansion of the $\mathrm{BV}^{+}$cell population in Eomes $\Delta$ Epi embryos at e7.5. d Nuclear Nanog staining in Eomes $\Delta$ Epi and control BV ${ }^{+}$embryos at e7.5. e Analysis of Ap2 $\gamma$, Sox2 and Stella in e7.5 control and Eomes $\Delta$ Epi BV ${ }^{+}$cells. $\mathbf{f}$ Brachyury staining in control and Eomes $\Delta$ Epi $\mathrm{BV}^{+}$e 7.5 embryos. All IF images are counter stained with DAPI. Scale bars $=100 \mu \mathrm{m}$

epiblast of gastrulating embryos ${ }^{15,20}$. Indeed, in addition to reduced PGC numbers in embryos lacking either Smad1 or Smad5, Smad1/5 double heterozygotes also have a reduced number of PGCs ${ }^{20}$, revealing a critical role for both Smad1 and Smad5 in germ cell development.

Taken together these experiments demonstrate that Smad1 functions in the epiblast during PGC specification, whereas in contrast Smad4 promotes PGC maintenance and/or migration. Hence, the Smad $4 \Delta$ Epi mutant phenotype is intermediate to the Smad $2 \Delta$ Epi and Smad1 $1^{-1-}$ phenotypes, as in Smad $2 \Delta$ Epi embryos Stella ${ }^{+}$cells form at the posterior side, while Smad1 mutants initiate pre-PGC differentiation but never develop specified PGCs.

Nodal and Bmp signalling requirements during PGCLC formation. Recent reports have described embryonic stem (ES) cell culture protocols for in vitro differentiation of PGCLCs that, as judged by gene-expression patterns and global epigenetic remodelling profiles, closely resemble bona fide $\mathrm{PGCs}^{7,8}$. To further investigate Nodal and Bmp signalling requirements we generated Smad2 ${ }^{-l-}$, Eomes $^{-l-}$, Smad1 ${ }^{-I-}$, Smad4 ${ }^{-I-}$ and $\mathrm{Wnt}^{-1-}$ ES 
a
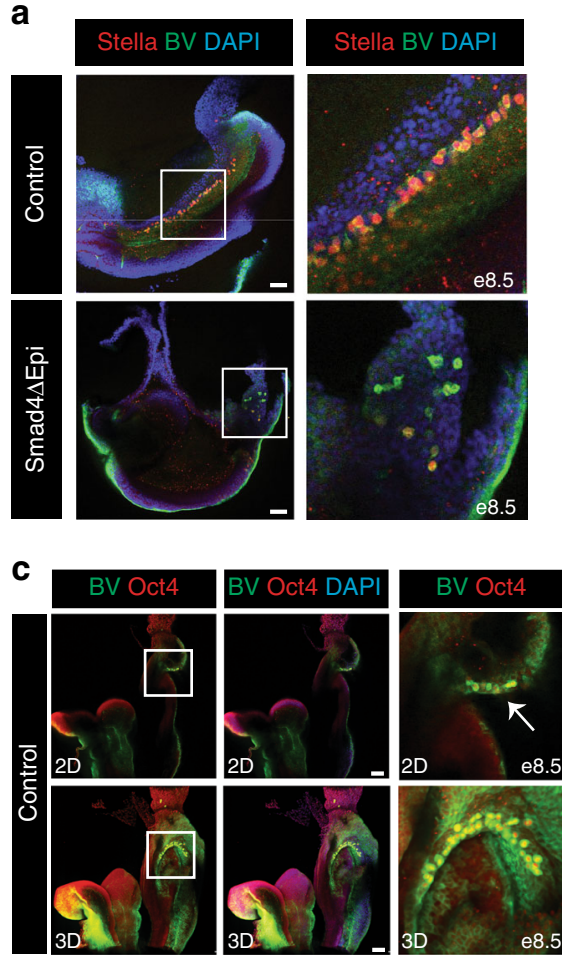

Fig. 3 Smad1 is required for PGC specification whereas Smad4 controls PGC maintenance and migration. a Stella staining at e8.5 shows PGCs migrating along the hindgut endoderm in $\mathrm{BV}^{+}$control but not Smad4 $\Delta$ Epi embryos. b Oct4 staining in Smad1 null and control e7.5 BV ${ }^{+}$embryos. c Single optical sections (2D) and z-stack projections (3D) showing Oct4 staining in the posterior side of Smad1 $\Delta$ Epi and control BV ${ }^{+}$embryos. Arrows indicate Oct4 and BV co-expressing cells. All IF images are counter stained with DAPI. Scale bars $=100 \mu \mathrm{m}$

cell lines carrying the BV transgene. Control and mutant $\mathrm{BV}^{+} \mathrm{ES}$ cells, grown under naïve conditions, were induced to form epiblast-like cells (EpiLCs), and subsequently aggregated under appropriate conditions to yield PGCLCs (Fig. 4a). As expected in wild-type cultures at day 2 and day 4 of PGCLC differentiation BV/ Stella, BV/Oct4, as well as BV/Ap2 $\gamma$ co-expressing cells were detectable by immunofluorescence (Fig. 4b, Supplementary Figure $4 \mathrm{~A}$ and $\mathrm{B}$ ). As a negative control, consistent with previous studies ${ }^{41}$, Wnt ${ }^{-1-}$ cultures contained only a few double positive PGCLCs (Fig. 4b, Supplementary Figure 4A and B). Our Smad2 ${ }^{-1-}$, Eomes $^{-l-}$, Smad1 $1^{-l-}$ and Smad4 ${ }^{-l-}$ ES cell lines yielded BV/Stella, BV/Oct4 and BV/Ap2 $\gamma$ double positive PGCLCs (Fig. 4b, Supplementary Figure $4 \mathrm{~A}$ and B). However, as judged by immunofluorescence and real-time quantitative polymerase chain reaction (RT-qPCR) analysis, the efficiency of PGCLC induction was compromised as compared to wild-type control cultures. Thus, Smad2 ${ }^{-1-}$, Eomes $^{-1-}$ and Smad4 ${ }^{-1-}$ cell aggregates express lower levels of $\operatorname{Prdm} 1$ (Blimp1) and Tfap2c (Ap2 $\gamma$ ), while Smad1 $1^{-1-}$ cultures show reduced Tfap $2 c$ and increased levels of the somatic marker Hoxb1 (Fig. 4c). These results demonstrate that in contrast to the situation in vivo, in cultures containing high levels of exogenously added growth factors, Eomes ${ }^{-1-}$ and Smad1-I- ES cells can differentiate into PGCLCs.

Decreased Bmp responsiveness accompanies PGC specification. At e6.5 p-Smad159 staining is normally restricted to the extra-embryonic VE, the anterior VE and the PPE whereas the posterior embryonic VE lacks p-Smad159 (Fig. 5a). However, in Smad2 $\Delta$ VE embryos, p-Smad159 is detectable throughout the entire VE at e5.5 (Fig. 1a), but slightly later at e6.5 the VE does not have any $\mathrm{p}$-Smad159 reactivity (Fig. 5a). The mesodermal cell population in e6.5 Smad2 $\triangle \mathrm{VE}$ embryos surrounding $\mathrm{BV}^{+}$cells
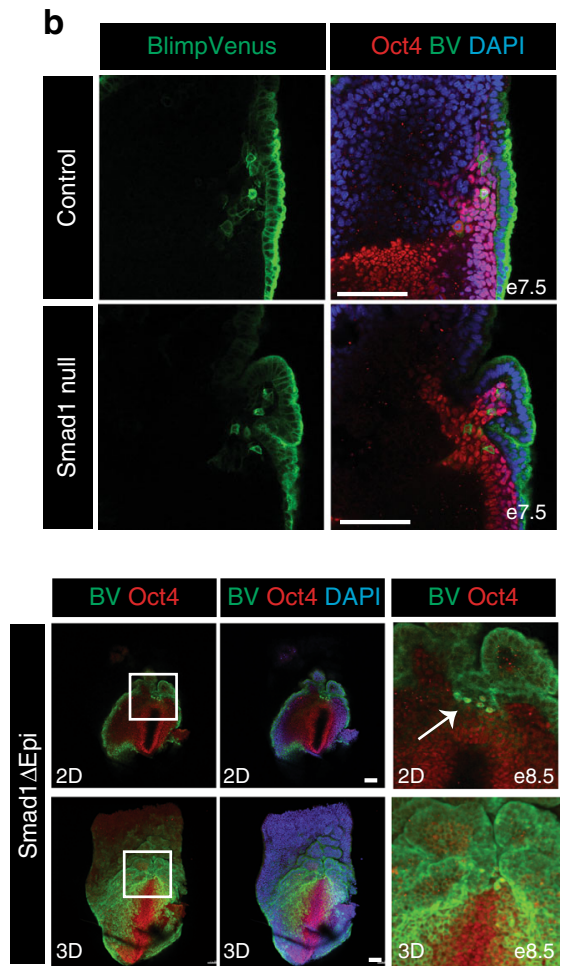

display strong p-Smad159 immunoreactivity. However, the $\mathrm{BV}^{+}$ cells themselves have substantially reduced levels of p-Smad159 staining (Fig. 5a). Likewise, the BV high-expressing cells in control e7.5 embryos, as well as the expanded numbers of $\mathrm{BV}^{+}$ cells present in e7.5 Smad2 $\Delta$ VE embryos, lack p-Smad159 reactivity (Fig. 5b). These results demonstrate that down-regulated pSmad159 levels distinguishes specified PGCs from their somatic neighbours and that specified PGCs do not respond to high levels of Bmp signalling. Additionally, Eomes $\Delta$ Epi embryos also display a high proportion of $\mathrm{BV}^{+}$epiblast cells lacking $\mathrm{p}$-Smad159 activity (Supplementary Figure 5A). Decreased p-Smad159 staining was also observed within the $\mathrm{BV}^{+}$cells generated during in vitro PGCLC differentiation (Fig. 5c, Supplementary Figure $5 \mathrm{~B}$ ). Thus, decreased responsiveness to $\mathrm{Bmp}$ signalling is a characteristic feature of PGC cell populations, in both in vitro and in vivo settings.

To directly assess whether Blimp1 expression influences Smad159 phosphorylation levels, we generated Blimp1-/embryos carrying the $\mathrm{BV}$ transgene. $\mathrm{BV}^{+}$epiblast cells were visible at the base of the allantoic bud at e7.5 in Blimp1-/embryos (Fig. 5d), strengthening the notion that Blimp1 functional activity is not required at early stages during prePGC formation. Interestingly a subset of $\mathrm{BV}^{+}$cells display decreased p-Smad159 levels, while others retain p-Smad159 reactivity (Fig. 5d). To test whether loss of p-Smad159 within the maturing PGCs potentially reflects reduced levels of transcription we examined RNA-seq data sets ${ }^{42,43}$ (Supplementary Table 3). Reduced Smad1 and Smad5 expression levels were reported in e7.5 PGCs as compared to the surrounding mesodermal cell population (Supplementary figure 5C). Moreover RNA-seq analysis of wild-type PGCs ${ }^{43}$ confirms that Smad1 expression is down-regulated during PGC development (Supplementary Figure $5 \mathrm{D})$, whereas in contrast $\mathrm{BV}^{+} \mathrm{Blimp}^{-/-}$PGCs fail to down- 
a

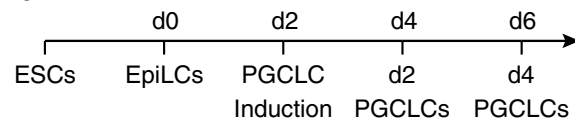

b

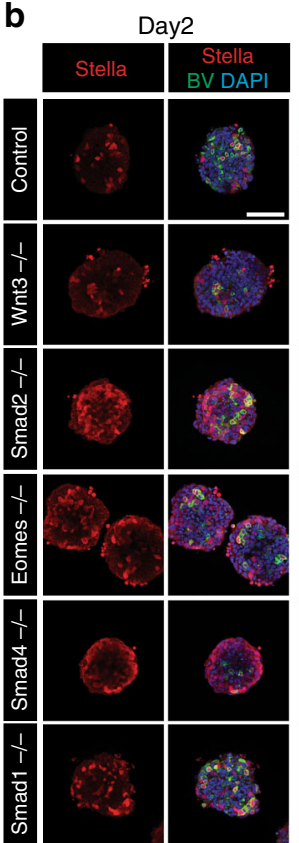

Day4

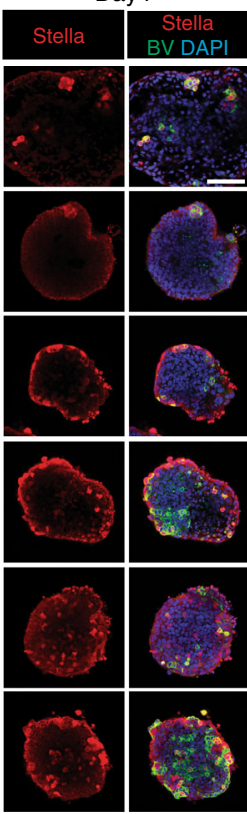

C $\operatorname{Prdm} 1$
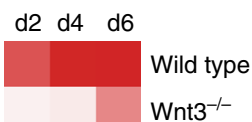

Smadi-l-

Eomes $^{-1-}$

Smad4 ${ }^{-1-}$

Smad1 ${ }^{-1-}$

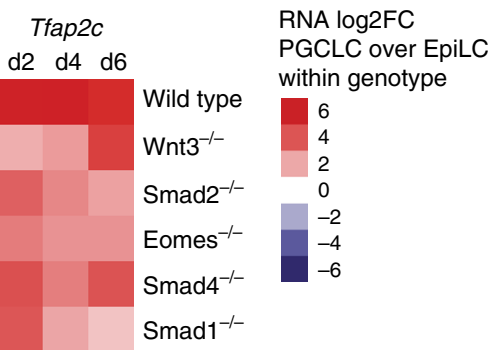

Hoxb1

d2 $d 4$ d6

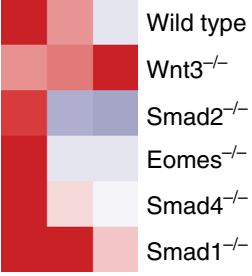

Fig. 4 Abilities of mutant ES cells lacking Nodal/Bmp/Smad pathway components to undergo PGCLC differentiation. a Time-line and culture protocol used for PGCLC differentiation. b Stella IF staining of day 2 and day 4 BV + PGCLC aggregates derived from the indicated genotypes, counter stained with DAPI. Scale bars $=100 \mu \mathrm{m}$. c Heatmap showing the log2 fold changes (log2FC) of Prdm1, Tfap2c and Hoxb1 expression, as judged by RT-qPCR, at day 2, 4 and 6 of PGCLC differentiation in control and mutant cells of the indicated genotypes, relative to EpiLCs

regulate Smad1 expression (Supplementary Figure 5D). Consistent with this recent Blimp1 ChIP-Seq experiments analysing d6 PGCLCs $^{43}$ (Supplementary Table 3) allowed us to identify a Blimp1 peak downstream of the Smad1 first coding exon containing the canonical Blimp1 binding motif (Fig. 5e). Collectively, these observations suggest that Blimp1 may directly repress Smad1 transcription during early PGC development. Conditional deletion of Blimp1 in mature PGCs, using the StellaCre deleter strain ${ }^{43}$, has no effect on Smad1 transcriptional levels (Supplementary Figure 5D). Thus, Blimp1 may down-regulate Smad1 expression at early stages of PGC development, potentially ensuring that the emerging PGCs are refractory to the high-Bmp signalling environment in the posterior epiblast that normally promotes development of the ExM.

\section{Discussion}

The transcriptional regulators and epigenetic machinery responsible for guiding allocation of the PGC lineage have been intensively investigated over the past decade ${ }^{1,5}$. The small population of prePGCs $(\sim 5)$ initially present at e6.25 in the proximal epiblast of prestreak embryos are marked by expression of the zinc finger transcriptional repressor Blimp1 $1^{3,44}$, together with the pluripotency factors Oct4 and Nanog. Slightly later at e6.75, Prdm14 expression is induced in developing PGCs ${ }^{45}$ and subsequently, coincident with formation of the posterior ExM, PGCs activate expression of Dppa3 (Stella), Tfap2c (Ap2 $\gamma$ ) and Sox2. The cluster of specified PGCs $(\sim 40)$ is detectable at e7.5 within the ExM at the base of the allantois. Over the following $24 \mathrm{~h}$ these committed PGCs migrate into the endodermal layer of the forming hindgut pocket.
Dose-dependent Nodal/Smad functional activities are known to be required for $\mathrm{A}-\mathrm{P}$ and left-right axis patterning in the early mouse embryo ${ }^{10}$. Functional contributions during gastrulation and within the PS derivatives have been extensively characterised $^{10}$. Here, we dissected Nodal/Bmp/Smad signalling cues within embryonic and extra-embryonic tissues that govern the PGC developmental programme. The present experiments now reveal importantly that Nodal/Smad2 activities also regulate allocation of the pre-PGC population. Thus Nodal null embryos prematurely initiate pre-PGC formation at e5.5. However, these $\mathrm{BV}^{+}$epiblast cells are ectopically positioned and lack Nanog and Ap2 $\gamma$ expression. Nanog, an important regulator of PGC development in vivo ${ }^{46,47}$, has been previously been shown to induce PGCLC differentiation in vitro ${ }^{48}$. Nanog directly upregulates both Prdm1 (Blimp1) and Prdm14 expression in PGCLCs ${ }^{48}$. However, over-expression activates $\operatorname{Prdm} 14$ expression prior to $\operatorname{Prdm} 1^{48}$ in EpiLCs, whereas $\operatorname{Prdm} 1$ expression in pre-PGCs in vivo precedes that of $\operatorname{Prdm} 14^{45,48}$. Thus Nanog re-expression downstream of Nodal seems to be non-essential for induction of Prdm1 expression per se. Rather Nanog may primarily function to activate Prdm14. The present results demonstrate that Nodal signalling in the proximal epiblast, prior to the onset of streak formation, is required to fully reactivate and maintain high levels of Nanog expression.

Nodal is transiently expressed in the proximal epiblast and expression decreases as the streak elongates ${ }^{49}$. The high levels retained in the anterior streak function to induce DE and midline fates ${ }^{10}$. Mutant embryos lacking expression of its downstream effector Smad2 fail to induce the antagonists Cer1 and Lefty1 in the VE and consequently display ectopic Nodal signalling 
a
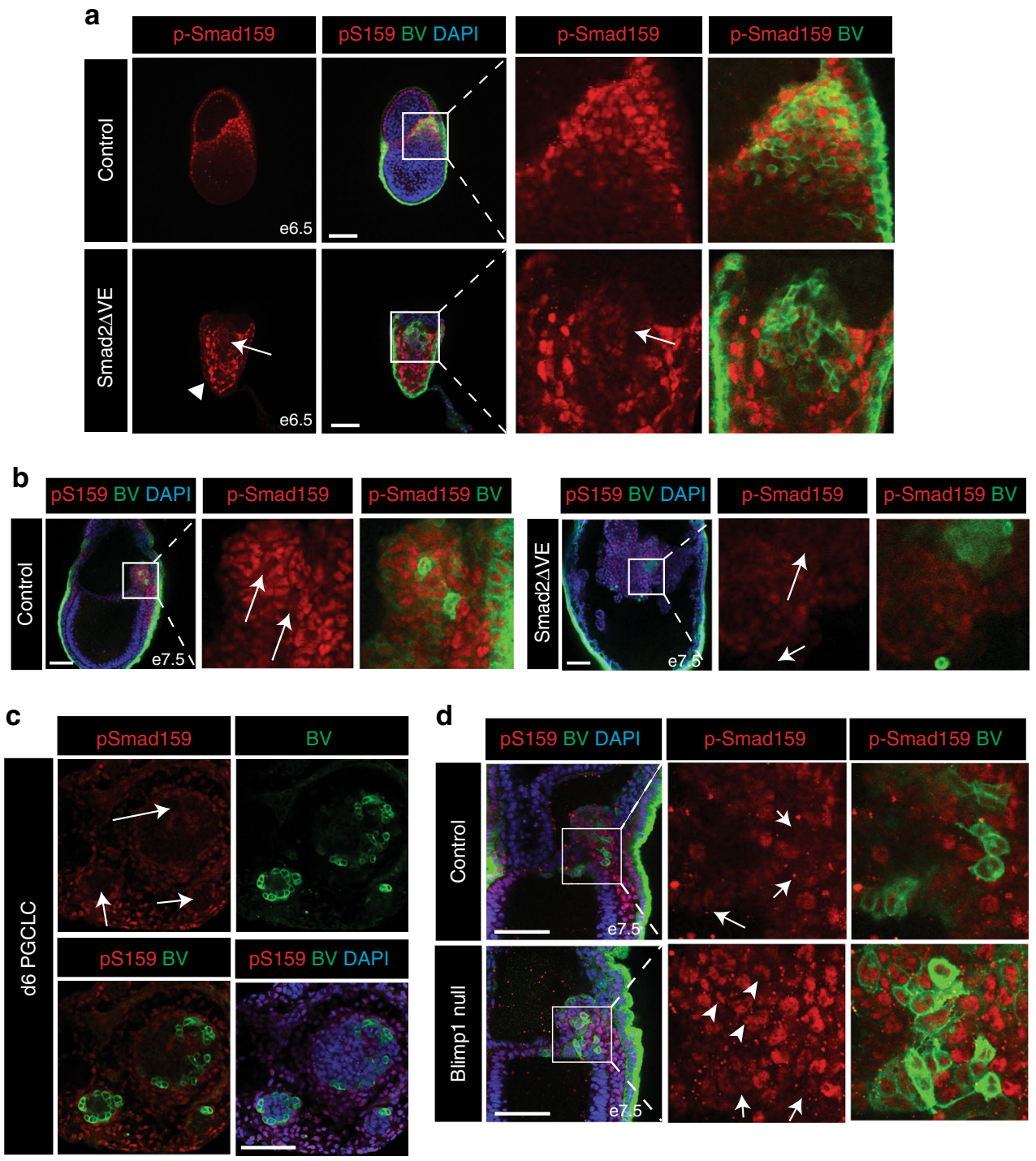

e GFP-Blimp1 ChIP-Seq (Mitani et al. 2017) [0-90]
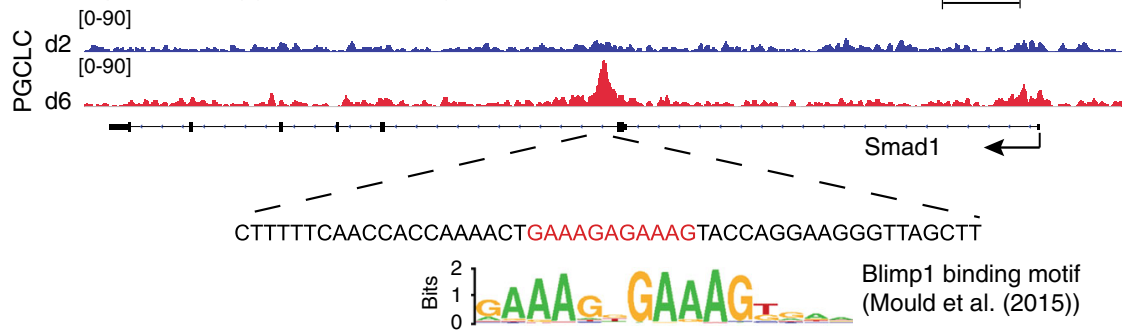

Fig. 5 PGCs down-regulate $\mathrm{p}-\mathrm{Smad159}$ to become refractory to the high-Bmp signalling environment. a $\mathrm{p}$-Smad159 staining of $\mathrm{BV}^{+}$cell populations. Arrowhead confirms e6.5 Smad2 $\Delta$ VE embryos lack p-Smad159 in the VE, while arrows indicates weak activity in BV-expressing epiblast cells. $\mathbf{b}$ p-Smad159 staining in control and Smad2 $\Delta$ VE BV ${ }^{+}$embryos at e7.5. Arrows indicate loss of p-Smad159 staining in BV high-expressing cells. c $p$-Smad159 staining in $\mathrm{BV}^{+}$wild-type d6 PGCLC aggregates. Arrows indicate BV+ cell populations lacking $\mathrm{p}$-Smad159 reactivity. d p-Smad159 staining in control and Blimp1-/$\mathrm{BV}^{+}$embryos at e7.5. Arrows indicate reduction of $\mathrm{p}$-Smad159 reactivity in BV high-expressing cells in expanded panels. Arrowheads indicate retained $\mathrm{p}-$ Smad159 activity in BV+ cells in the Blimp1 ${ }^{-/-}$mutant embryo. All IF images are counter stained with DAPI. Scale bars $=100 \mu \mathrm{m}$. e Genome browser track view of GFP-ChIP wiggle plots from GFP-Blimp1 expressing PGCLC cultures at day (d)2 (blue) and d6 (red) showing enrichment of GFP-Blimp1 density at a Smad1 intronic region. The sequence below the GFP-Blimp1 ChIP site contains a Blimp1 binding motif indicated in red. The consensus Blimp1 binding motif previously identified by genome-wide ChIP-seq data sets is also indicated 65

throughout the entire epiblast ${ }^{12,25}$. The present experiments demonstrate that conditional Smad $2 \Delta$ VE mutants phenocopy Smad2 null embryos. As judged by down-regulated expression of Otx2 and Sox2, together with up-regulated Brachyury expression, the mutant epiblasts appear to prematurely adopt a proximal posterior character. An inverse relationship between p-Smad159 and p-Smad23 signalling in the VE has previously been characterised $^{9}$. Here, we found that e5.5 Smad $2 \Delta \mathrm{VE}$ embryos display ectopic p-Smad159 activation throughout the VE. Interestingly, these disturbances in $\operatorname{Smad} 2 \Delta \mathrm{VE}$ mutants are associated with 
decreased expression of the Bmp antagonist Gdf3 and likely accounts for the increased levels of p-Smad159 in the VE. Thus imbalanced Nodal/Bmp signalling leads to the precocious appearance and markedly increased numbers of pre-PGCs at e6.5. These results demonstrate Nodal/Smad2 signals localised to the proximal epiblast govern competency to acquire PGC characteristics and promote the establishment of the PGC niche.

Recent work suggests that occupancy at a putative T-box site, $10 \mathrm{~kb}$ upstream of the Prdm1 transcription start site, by the T-box transcription factor family member Brachyury activates Blimp1 expression in the pre-PGC population ${ }^{41}$. Thus Brachyury mutant embryos activate but fail to maintain $\mathrm{BV}^{+}$pre-PGCs ${ }^{41}$. Consistent with this, forced expression of either Brachyury or Eomes in EpiLCs leads to up-regulated $\operatorname{Prdm} 1$ expression ${ }^{41}$, suggesting that Eomes and Brachyury may function redundantly to activate $\operatorname{Prdm} 1$ expression. However, the present experiments demonstrate that Eomes is dispensable for Blimp1 induction in the epiblast. Moreover, the $\mathrm{BV}^{+}$cells formed in the absence of Eomes mostly lack Brachyury expression, strongly suggesting that Brachyury and Eomes are dispensable for initial induction of Blimp1.

Eomes in the early PS directly activates Mesp1 expression necessary to allow nascent mesoderm to down-regulate E-Cadherin and undergo EMT ${ }^{36,50}$. Here, we demonstrate that Eomes, acting immediately downstream of Nodal within the epiblast ${ }^{37}$, plays an essential role during the early stages of PGC formation. In the absence of Eomes, we observe an expanded population of $\mathrm{BV}^{+}$cells in the proximal epiblast. However, these pre-PGCLCs lack Nanog expression and fail to activate the germ cell programme. Interestingly, Eomes mutant $\mathrm{BV}^{+}$cells, in close proximity to Bmp4 signals from the overlying ExE, lack p-Smad159 activity and are refractory to Bmp signalling. The absence of Eomes in the proximal epiblast disturbs formation of the PGC niche within the posterior ExM population that normally promotes PGC specification and survival ${ }^{51,52}$. Previous work shows that Eomes null PS cells can undergo EMT and generate mesodermal cell populations in vitro ${ }^{36}$. Here, we demonstrate that Eomes null ES cells can differentiate into BV/Oct4/Ap2 $\gamma /$ Stella positive PGCLCs, albeit at a lower efficiency as compared to wildtype controls, most likely due to their ability to generate a supporting mesodermal population. Thus, Eomes is not intrinsically required for Blimp1 induction and PGC formation per se. Rather, acting downstream of Nodal, Eomes is essential to promote formation of the posterior signalling niche necessary for maturation of the PGC lineage.

How PGCs maintain their unique cell-type identity within the predominant mesodermal signalling environment at the base of the allantois remains mysterious. Here, we describe an unappreciated cellular mechanism that allows PGCs to insulate themselves and become refractory to Bmp signals. Thus, we found at e7.5 that $\mathrm{BV}^{+}$cells display reduced Smad159 phosphorylation levels. Moreover within PGCLC aggregates p-Smad159 activity and BV expression are mutually exclusive. Down-regulated expression of both Smad1 and Smad5 was previously observed in PGCs compared to the surrounding mesoderm $^{42}$. Blimp1 may directly repress Smad1 transcription via occupancy at a site within the Smad1 locus. Consistent with this idea recent RNA-seq data sets ${ }^{43}$ reveal that Smad1 expression in Blimp1-deficient pre-PGCs is not down-regulated.

The cluster of PGCs at the base of the allantois, that themselves lack Bmp4 expression, are surrounded by ExM expressing high levels of Bmp4 ${ }^{17}$. While PGCs themselves become refractory to the Bmp signalling environment, their survival and further development is thought to be critically dependent on the ability of the adjacent ExM to provide extrinsic trophic growth factors ${ }^{52}$. Recent in vitro experiments similarly suggest that the role of Smad1 signalling during PGCLC differentiation may be to generate somatic cells that function to promote PGCLC survi$\mathrm{val}^{53}$. Likewise, we observe here in PGCLC aggregates localised pockets of $\mathrm{BV}^{+}$cells within $\mathrm{p}-\mathrm{Smad} 159$ active regions. Thus, a continuum of Bmp signalling in the early post-implantation embryo is required to induce the formation of pre-PGCs in the early epiblast and subsequently at later stages establishes the posterior signalling niche necessary for PGC specification.

Imaging studies demonstrate that PGCs actively migrate as individual cells from the base of the allantois towards the overlying endoderm ${ }^{54}$. However, the molecular pathways guiding this initial directional migration remain ill-defined. Besides intrinsic regulators of cell motility the posterior endoderm also plays a critical role guiding PGC migration. For example, in Sox17 mutant embryos that display defective endoderm formation, PGCs are formed, mature and initiate migration towards the endoderm but lack the ability to appropriately integrate to the endoderm ${ }^{55}$. Similarly we found here in Smad $2 \Delta \mathrm{VE}$ and Smad4 $\Delta$ Epi embryos that PGCs are appropriately specified, but fail to migrate towards the endoderm. PGCs normally extend long filopodia-like structures at e7.5 $5^{54}$. In contrast in Smad2 $\Delta$ VE embryos endoderm formation is compromised, filopodia-like structures are never observed and PGCs remain as clusters of epithelial-like cells. Likewise, Smad4 $\Delta$ Epi embryos fail to form $\mathrm{DE}^{39}$. Migration defects could potentially reflect the intrinsic loss of Smad4-dependent PGC functional activities, or alternatively, defective endoderm formation potentially results in the failure to produce chemo-attractants. The extracellular matrix (ECM) also plays a crucial role in guiding PGC migration ${ }^{56,57}$. We previously reported that Smad4 controls ECM deposition in early developmental stages ${ }^{58}$. Further studies on the trophic signals emitted from the posterior endoderm and the potential role of Smad4dependent signalling in ECM composition will be required to further define Bmp/Nodal Smad requirements during PGC migration.

An antagonistic relationship between Nodal and Bmp pathways has been described in a variety of developmental contexts including within the VE for establishing initial proximal-distal polarity, patterning of the PS, morphogenesis of the amnion and correct establishment of the left-right body plan $9,10,13,59,60$. The present experiments further demonstrate that these regulatory cues govern cell fate decisions in the posterior epiblast causing a discrete subpopulation to adopt a germ cell versus somatic cell fate. Importantly, we found that Blimp1 induction within the PGCs is associated with down-regulated pSmad159 levels providing a cell intrinsic regulatory mechanism that allows this discrete subpopulation to become non-responsive to local Bmp signalling cues. Future experiments will be needed to further define dynamic cellular events controlling this developmental switch.

\section{Methods}

Animal care. Smad2 ${ }^{-1-25}$, Smad2CA 61, Nodal $^{-1-24}$, Eomes $^{-1-}$, Eomes ${ }^{C A} 36$, Smad4 ${ }^{-1-}, S_{m a d 4}{ }^{C A} 39, \operatorname{Prdm} 1^{-1-4}, \operatorname{Prdm} 1^{C A}{ }^{62}, \operatorname{Smad1}^{-1-}, \operatorname{Smad1}^{C A}{ }^{15}$, Prdm1 $1_{\text {Cre-Lacz 40 }}$,Ttr-Cre ${ }^{26}$, Sox2-Cre ${ }^{33}$, Prdm1-mVenus ${ }^{32}$ alleles were genotyped as described. Supplementary Table 2 indicates inter-crosses produced for this study and acronyms used for embryos. All animal experiments were performed in accordance with Home Office (UK) regulations and approved by the University of Oxford Local Ethical Committee.

The sex of the individual embryos was not determined-over the course of the experiments we assume that the ratio of males to females is $1: 1$ reflecting the sex ratio of the pups at birth.

Generation of knockout ES cell lines and ES cell culture. Blastocysts for ESC derivation were obtained from inter-crosses of $W n t 3^{+/-23}, \operatorname{Smad1} 1^{+/-15}$, Smad2 $2^{+/-25}$, Smad4 ${ }^{+/-} 39$ and Eomes ${ }^{+/-} 36$ mice harbouring the Prdm1-mVenus BAC transgene ${ }^{32}$. All ESC lines used were grown in feeder-free conditions on $0.1 \%$ gelatin-coated dishes at $6 \% \mathrm{CO}_{2}$ at $37^{\circ} \mathrm{C}$. ESCs were cultured in serum-free media 
containing N2B27 (Takara, Y40002) supplemented with $1 \mu \mathrm{M}$ PD0325091 and $3 \mu \mathrm{M}$ CHIR99021 and $1000 \mathrm{U} / \mathrm{ml} \mathrm{LIF}$.

PGCLC cultures. EpiLCs and PGCLCs were induced as previously described ${ }^{63}$. In brief, $7 \times 10^{5}$ ESCs were washed and resuspended in N2B27 medium (Takara, Cat\#Y40002) supplemented with $12 \mathrm{ng} / \mathrm{ml} \mathrm{bFgf} \mathrm{(Invitrogen,} \mathrm{13256-029),} 20 \mathrm{ng} / \mathrm{ml}$ Activin A and 1\% KSR (Gibco, 10828, Lot:1508151) and grown on fibronectincoated $\left(10 \mu \mathrm{g} / \mathrm{cm}^{2}\right.$ ) (Millipore, FC010) $6 \mathrm{~cm}$ dishes for $48 \mathrm{~h}$ to form EpiLCs. 2000 EpiLCs were then washed and plated into ultra-low attachment U-bottom shaped 96-well plates in serum-free medium (GK15; GMEM (Invitrogen) with 15\% KSR, $0.1 \mathrm{mM}$ NEAA, $1 \mathrm{mM}$ sodium pyruvate, $0.1 \mathrm{mM} 2$-mercaptoethanol, $100 \mathrm{U} / \mathrm{ml}$ penicillin, $0.1 \mathrm{mg} / \mathrm{ml}$ streptomycin, and $2 \mathrm{mM} \mathrm{L-glutamine)} \mathrm{in} \mathrm{the} \mathrm{presence} \mathrm{of} \mathrm{the}$ cytokines BMP4 (500 ng/ml; R\&D Systems, 314-BP), LIF (1000 U/ml; Millipore, ESG1107), SCF (100 ng/ml; R\&D Systems, 455-MC), BMP8b (500 ng/ml; R\&D Systems, 1073-BP) and EGF (50 ng/ml; R\&D Systems, 2028-EG). Differentiations were performed in two independent clonal cell lines per genotype.

Immunofluorescence. d2 and d4 PGCLCs as well as E5.5-E8.5 mouse embryos were harvested, washed in phosphate-buffered saline (PBS) and fixed in $1 \%$ paraformaldehyde (PFA) o/n at $4{ }^{\circ} \mathrm{C}$. After three washes in PBS containing $0.1 \%$ Triton X-100 (PBS-Tx), samples were permeabilised in PBS containing $0.5 \%$ Triton $\mathrm{X}-100$ followed by three washes in PBS-Tx, blocked in 5\% donkey serum and $0.2 \%$ BSA in PBS-Tx for $1 \mathrm{~h}$ at RT and incubated overnight with primary antibodies in blocking solution at $4{ }^{\circ} \mathrm{C}$. Following four washes in PBS-Tx, samples were incubated with fluorophore-conjugated secondary antibodies in blocking solution $(2 \mathrm{~h}$, RT) followed by three-five washes in PBS-Tx, one wash in PBS-Tx containing $2 \mu \mathrm{g} /$ $\mathrm{ml}$ DAPI and three washes in PBS-Tx prior to mounting in Vectashield with DAPI (H-1200). Samples were imaged the following day on an Olympus Fluoview FV1000 confocal microscope and image data was processed using ImageJ and Bitplane Imaris software. Antibodies are listed in the Supplementary Table 3. Multiple litters of each intercross were examined, with a minimum of $n=3$ mutant embryos stained and imaged.

RT-qPCR. Two hundred and fifty nanogram RNA was reverse transcribed to cDNA using Superscript III First Strand Synthesis System (Life Technologies, Cat\#18080-051) and diluted to $100 \mu \mathrm{l}$ final volume in $\mathrm{H}_{2} \mathrm{O}(2.5 \mathrm{ng} / \mathrm{ul})$. Two microlitre (5 ng) cDNA were used per RT-qPCR reaction in duplicate using SYBRgreen kit (Qiagen, Cat\#204143). Relative gene expression was normalised to Gapdh expression and calculated as $2^{\Delta \Delta} \mathrm{Ct}$. Average expression levels were calculated for two technical replicates from two independent cell lines per genotype. RT-qPCR primer sequences are listed in Supplementary Table 4.

In situ hybridisation and immunohistochemistry. Whole-mount in situ hybridisation analysis was performed as befor $\mathrm{e}^{50}$, using antisense riboprobes for $B m p 2^{64}$, $B m p 4^{13}$ and $G d f 3^{28}$. For Blimpl immunohistochemistry, E7.5 decidua were fixed overnight in $4 \%$ PFA, dehydrated in ethanol, embedded in paraffin wax and sectioned $(6 \mu \mathrm{m})$. Dewaxed sections were subjected to antigen retrieval by boiling for $1 \mathrm{~h}$ in Tris/EDTA ( $\mathrm{pH} \mathrm{9.0)}$ and permeabilized for $10 \mathrm{~min}$ in $0.1 \%$ Triton X-100 in TBS. Sections were subsequently blocked with $10 \%$ normal goat serum in TBS. Sections were incubated in rat monoclonal anti-Blimp1 (1:500 dilution, sc-130917, Santa Cruz Biotechnology) in block overnight at $4{ }^{\circ} \mathrm{C}$ and signal-amplified with rabbit anti-rat secondary antibody (AI-4001, Vector Laboratories) for $45 \mathrm{~min}$ at RT followed by peroxidase blocking for $20 \mathrm{~min}$ at RT and development with Envison System-HRP for rabbit antibodies (K4011, DAKO) and Vector Red substrate (SK4805 , Vector Laboratories). Sections were lightly counter stained with haematoxylin, coverslipped and imaged.

Haematoxylin and eosin staining was performed as previously described ${ }^{36}$.

Reporting summary. Further information on experimental design is available in the Nature Research Reporting Summary linked to this article.

\section{Data availability}

The authors declare that all data supporting the findings of this study are available within the article and its supplementary information files or from the corresponding author upon reasonable request. Publicly available source data in Fig. 5 and Supplementary Figure 5 were obtained from GSE91041, GSE91040 and E-MTAB-1178.

Received: 2 October 2018 Accepted: 13 February 2019

Published online: 06 March 2019

\section{References}

1. Saitou, M. \& Yamaji, M. Primordial germ cells in mice. Cold Spring Harb Perspect Biol 4, https://doi.org/10.1101/cshperspect.a008375 (2012).
2. Lawson, K. A. \& Hage, W. J. Clonal analysis of the origin of primordial germ cells in the mouse. Ciba Found. Symp. 182, 68-84 (1994).

3. Ohinata, Y. et al. Blimp1 is a critical determinant of the germ cell lineage in mice. Nature 436, 207-213 (2005)

4. Vincent, S. D. et al. The zinc finger transcriptional repressor Blimp1/Prdm1 is dispensable for early axis formation but is required for specification of primordial germ cells in the mouse. Development 132, 1315-1325 (2005).

5. Magnusdottir, E. \& Surani, M. A. How to make a primordial germ cell. Development 141, 245-252 (2014).

6. Saitou, M., Kagiwada, S. \& Kurimoto, K. Epigenetic reprogramming in mouse pre-implantation development and primordial germ cells. Development 139, 15-31 (2012).

7. Hayashi, K., Ohta, H., Kurimoto, K., Aramaki, S. \& Saitou, M. Reconstitution of the mouse germ cell specification pathway in culture by pluripotent stem cells. Cell 146, 519-532 (2011).

8. Kurimoto, K. et al. Quantitative dynamics of chromatin remodeling during germ cell specification from mouse embryonic stem cells. Cell Stem Cell 16, 517-532 (2015).

9. Yamamoto, M. et al. Antagonism between Smad1 and Smad2 signaling determines the site of distal visceral endoderm formation in the mouse embryo. J. Cell Biol. 184, 323-334 (2009).

10. Robertson, E. J. Dose-dependent Nodal/Smad signals pattern the early mouse embryo. Semin Cell Dev. Biol. 32, 73-79 (2014).

11. Massague, J. TGF-beta signaling in development and disease. FEBS Lett. 586, 1833 (2012)

12. Brennan, J. et al. Nodal signalling in the epiblast patterns the early mouse embryo. Nature 411, 965-969 (2001).

13. Winnier, G., Blessing, M., Labosky, P. A. \& Hogan, B. L. Bone morphogenetic protein-4 is required for mesoderm formation and patterning in the mouse. Genes Dev. 9, 2105-2116 (1995).

14. Dunn, N. R., Vincent, S. D., Oxburgh, L., Robertson, E. J. \& Bikoff, E. K. Combinatorial activities of Smad2 and Smad3 regulate mesoderm formation and patterning in the mouse embryo. Development 131, 1717-1728 (2004).

15. Tremblay, K. D., Dunn, N. R. \& Robertson, E. J. Mouse embryos lacking Smad1 signals display defects in extra-embryonic tissues and germ cell formation. Development 128, 3609-3621 (2001).

16. Chang, $H$. et al. Smad5 knockout mice die at mid-gestation due to multiple embryonic and extraembryonic defects. Development 126, 1631-1642 (1999).

17. Lawson, K. A. et al. Bmp4 is required for the generation of primordial germ cells in the mouse embryo. Genes Dev. 13, 424-436 (1999).

18. Ying, Y., Liu, X. M., Marble, A., Lawson, K. A. \& Zhao, G. Q. Requirement of Bmp8b for the generation of primordial germ cells in the mouse. Mol. Endocrinol. 14, 1053-1063 (2000).

19. Ying, Y. \& Zhao, G. Q. Cooperation of endoderm-derived BMP2 and extraembryonic ectoderm-derived BMP4 in primordial germ cell generation in the mouse. Dev. Biol. 232, 484-492 (2001).

20. Arnold, S. J., Maretto, S., Islam, A., Bikoff, E. K. \& Robertson, E. J. Dosedependent Smad1, Smad5 and Smad8 signaling in the early mouse embryo. Dev. Biol. 296, 104-118 (2006).

21. Ohinata, Y. et al. A signaling principle for the specification of the germ cell lineage in mice. Cell 137, 571-584 (2009).

22. Ben-Haim, N. et al. The Nodal precursor acting via activin receptors induces mesoderm by maintaining a source of its convertases and BMP4. Dev. Cell 11, 313-323 (2006).

23. Liu, P. et al. Requirement for Wnt3 in vertebrate axis formation. Nat. Genet 22, 361-365 (1999)

24. Conlon, F. L., Barth, K. S. \& Robertson, E. J. A novel retrovirally induced embryonic lethal mutation in the mouse: assessment of the developmental fate of embryonic stem cells homozygous for the 413.d proviral integration. Development 111, 969-981 (1991).

25. Waldrip, W. R., Bikoff, E. K., Hoodless, P. A., Wrana, J. L. \& Robertson, E. J. Smad2 signaling in extraembryonic tissues determines anterior-posterior polarity of the early mouse embryo. Cell 92, 797-808 (1998).

26. Kwon, G. S. \& Hadjantonakis, A. K. Transthyretin mouse transgenes direct RFP expression or Cre-mediated recombination throughout the visceral endoderm. Genesis 47, 447-455 (2009).

27. Levine, A. J. \& Brivanlou, A. H. GDF3, a BMP inhibitor, regulates cell fate in stem cells and early embryos. Development 133, 209-216 (2006).

28. Chen, C. et al. The Vg1-related protein Gdf3 acts in a Nodal signaling pathway in the pre-gastrulation mouse embryo. Development 133, 319-329 (2006).

29. Andersson, O., Bertolino, P. \& Ibanez, C. F. Distinct and cooperative roles of mammalian Vg1 homologs GDF1 and GDF3 during early embryonic development. Dev. Biol. 311, 500-511 (2007).

30. Hart, A. H., Hartley, L., Ibrahim, M. \& Robb, L. Identification, cloning and expression analysis of the pluripotency promoting Nanog genes in mouse and human. Dev. Dyn. 230, 187-198 (2004).

31. Yamaguchi, S., Kimura, H., Tada, M., Nakatsuji, N. \& Tada, T. Nanog expression in mouse germ cell development. Gene Expr. Patterns 5, 639-646 (2005). 
32. Ohinata, Y., Sano, M., Shigeta, M., Yamanaka, K. \& Saitou, M. A comprehensive, non-invasive visualization of primordial germ cell development in mice by the Prdm1-mVenus and Dppa3-ECFP double transgenic reporter. Reproduction 136, 503-514 (2008).

33. Hayashi, S., Lewis, P., Pevny, L. \& McMahon, A. P. Efficient gene modulation in mouse epiblast using a Sox2Cre transgenic mouse strain. Mech. Dev. 119 (Suppl 1), S97-S101 (2002).

34. Mulas, C., Kalkan, T. \& Smith, A. Nodal secures pluripotency upon embryonic stem cell progression from the ground state. Stem Cell Rep. 9, 77-91 (2017).

35. Senft, A. D. et al. Combinatorial Smad $2 / 3$ activities downstream of Nodal signaling maintain embryonic/extra-embryonic cell identities during lineage priming. Cell Rep. 24, 1977-1985 e1977 (2018).

36. Arnold, S. J., Hofmann, U. K., Bikoff, E. K. \& Robertson, E. J. Pivotal roles for eomesodermin during axis formation, epithelium-to-mesenchyme transition and endoderm specification in the mouse. Development 135, 501-511 (2008).

37. Simon, C. S. et al. Functional characterisation of cis-regulatory elements governing dynamic Eomes expression in the early mouse embryo. Development 144, 1249-1260 (2017).

38. Nowotschin, S. et al. The T-box transcription factor Eomesodermin is essential for AVE induction in the mouse embryo. Genes Dev. 27, 997-1002 (2013).

39. Chu, G. C., Dunn, N. R., Anderson, D. C., Oxburgh, L. \& Robertson, E. J. Differential requirements for Smad4 in TGFbeta-dependent patterning of the early mouse embryo. Development 131, 3501-3512 (2004).

40. Mould, A., Morgan, M. A., Li, L., Bikoff, E. K. \& Robertson, E. J. Blimp1/ Prdml governs terminal differentiation of endovascular trophoblast giant cells and defines multipotent progenitors in the developing placenta. Genes Dev. 26, 2063-2074 (2012).

41. Aramaki, S. et al. A mesodermal factor, T, specifies mouse germ cell fate by directly activating germline determinants. Dev. Cell 27, 516-529 (2013).

42. Magnusdottir, E. et al. A tripartite transcription factor network regulates primordial germ cell specification in mice. Nat. Cell Biol. 15, 905-915 (2013).

43. Mitani, T. et al. Principles for the regulation of multiple developmental pathways by a versatile transcriptional factor, BLIMP1. Nucleic Acids Res. 45, 12152-12169 (2017).

44. Saitou, M., Payer, B., O'Carroll, D., Ohinata, Y. \& Surani, M. A. Blimp1 and the emergence of the germ line during development in the mouse. Cell Cycle 4, 1736-1740 (2005).

45. Yamaji, M. et al. Critical function of Prdm14 for the establishment of the germ cell lineage in mice. Nat. Genet. 40, 1016-1022 (2008).

46. Chambers, I. et al. Nanog safeguards pluripotency and mediates germline development. Nature 450, 1230-1234 (2007).

47. Zhang, M. et al. Esrrb complementation rescues development of Nanog-null germ cells. Cell Rep. 22, 332-339 (2018).

48. Murakami, K. et al. Nanog alone induces germ cells in primed epiblast in vitro by activation of enhancers. Nature 529, 403-407 (2016).

49. Varlet, I., Collignon, J. \& Robertson, E. J. Nodal expression in the primitive endoderm is required for specification of the anterior axis during mouse gastrulation. Development 124, 1033-1044 (1997).

50. Costello, I. et al. The T-box transcription factor Eomesodermin acts upstream of Mesp1 to specify cardiac mesoderm during mouse gastrulation. Nat. Cell Biol. 13, 1084-1091 (2011).

51. de Sousa Lopes, S. M., Hayashi, K. \& Surani, M. A. Proximal visceral endoderm and extraembryonic ectoderm regulate the formation of primordial germ cell precursors. BMC Dev. Biol. 7, 140 (2007).

52. Fujiwara, T., Dunn, N. R. \& Hogan, B. L. Bone morphogenetic protein 4 in the extraembryonic mesoderm is required for allantois development and the localization and survival of primordial germ cells in the mouse. Proc. Natl Acad. Sci. USA 98, 13739-13744 (2001).

53. Mochizuki, K. et al. Repression of somatic genes by selective recruitment of HDAC3 by BLIMP1 Is essential for mouse primordial germ cell fate determination. Cell Rep. 24, 2682-2693 e2686 (2018).

54. Anderson, R., Copeland, T. K., Scholer, H., Heasman, J. \& Wylie, C. The onset of germ cell migration in the mouse embryo. Mech. Dev. 91, 61-68 (2000).

55. Hara, K. et al. Evidence for crucial role of hindgut expansion in directing proper migration of primordial germ cells in mouse early embryogenesis. Dev. Biol. 330, 427-439 (2009).

56. Garcia-Castro, M. I., Anderson, R., Heasman, J. \& Wylie, C. Interactions between germ cells and extracellular matrix glycoproteins during migration and gonad assembly in the mouse embryo. J. Cell Biol. 138, 471-480 (1997).
57. Richardson, B. E. \& Lehmann, R. Mechanisms guiding primordial germ cell migration: strategies from different organisms. Nat. Rev. Mol. Cell Biol. 11, 37-49 (2010).

58. Costello, I., Biondi, C. A., Taylor, J. M., Bikoff, E. K. \& Robertson, E. J. Smad4 dependent pathways control basement membrane deposition and endodermal cell migration at early stages of mouse development. BMC Dev. Biol. 9, 54 (2009).

59. Furtado, M. B. et al. BMP/SMAD1 signaling sets a threshold for the left/righ pathway in lateral plate mesoderm and limits availability of SMAD4. Genes Dev. 22, 3037-3049 (2008).

60. Pereira, P. N. et al. Antagonism of Nodal signaling by BMP/Smad5 prevents ectopic primitive streak formation in the mouse amnion. Development 139, 3343-3354 (2012)

61. Vincent, S. D., Dunn, N. R., Hayashi, S., Norris, D. P. \& Robertson, E. J. Cell fate decisions within the mouse organizer are governed by graded Nodal signals. Genes Dev. 17, 1646-1662 (2003).

62. Shapiro-Shelef, M. et al. Blimp-1 is required for the formation of immunoglobulin secreting plasma cells and pre-plasma memory B cells. Immunity 19, 607-620 (2003).

63. Hayashi, K. \& Saitou, M. Generation of eggs from mouse embryonic stem cells and induced pluripotent stem cells. Nat. Protoc. 8, 1513-1524 (2013).

64. Zhang, H. \& Bradley, A. Mice deficient for BMP2 are nonviable and have defects in amnion/chorion and cardiac development. Development 122, 2977-2986 (1996).

65. Mould, A. W., Morgan, M. A., Nelson, A. C., Bikoff, E. K. \& Robertson, E. J. Blimp1/Prdm1 functions in opposition to irf1 to maintain neonatal tolerance during postnatal intestinal maturation. PLoS Genet. 11, e1005375 (2015).

\section{Acknowledgements}

We would like to thank Mitinouri Saitou for generously providing the Blimp1-mVenus BAC transgenic mouse strain. Confocal microscopy was carried out in the Micron Advanced Bioimaging Unit (funded by Wellcome Trust Strategic Award 107457). This work was funded by the Wellcome Trust (099840/Z/12/A to A.D.S. and 102811/Z/13/2 to E.J.R.). E.J.R. is a Wellcome Trust Principal Research Fellow.

\section{Author contributions}

All the authors designed the experiments, A.D.S. and I.C. performed the experiments. A.D.S., I.C., E.K.B and E.J.R contributed to writing the paper.

\section{Additional information}

Supplementary Information accompanies this paper at https://doi.org/10.1038/s41467019-09052-w.

Competing interests: The authors declare no competing interests.

Reprints and permission information is available online at http://npg.nature.com/ reprintsandpermissions/

Journal peer review information: Nature Communications thanks Hiroshi Hamada and the other anonymous reviewer(s) for their contribution to the peer review of this work. Peer reviewer reports are available.

Publisher's note: Springer Nature remains neutral with regard to jurisdictional claims in published maps and institutional affiliations.

cc) (i) Open Access This article is licensed under a Creative Commons By Attribution 4.0 International License, which permits use, sharing, adaptation, distribution and reproduction in any medium or format, as long as you give appropriate credit to the original author(s) and the source, provide a link to the Creative Commons license, and indicate if changes were made. The images or other third party material in this article are included in the article's Creative Commons license, unless indicated otherwise in a credit line to the material. If material is not included in the article's Creative Commons license and your intended use is not permitted by statutory regulation or exceeds the permitted use, you will need to obtain permission directly from the copyright holder. To view a copy of this license, visit http://creativecommons.org/ licenses/by/4.0/.

(c) The Author(s) 2019 